\title{
Role of the host polymer matrix in light emission processes in nano-CdS/poly vinyl alcohol composite
}

\author{
G.Yu. Rudko ${ }^{\text {a,* }}$, A.O. Kovalchuk ${ }^{\text {a }}$, V.I. Fediv ${ }^{\text {b }}$, Q. Ren ${ }^{\text {c }}$, W.M. Chen ${ }^{\text {c }}$, I.A. Buyanova ${ }^{c}$, G. Pozina ${ }^{\mathrm{c}}$ \\ ${ }^{a}$ V. Lashkaryov Institute of Semiconductor Physics of National Academy of Sciences of Ukraine, 45, Pr. Nauky, Kiev, 03028, Ukraine \\ ${ }^{b}$ Department of Biophysics and Medical Informatics, Bukovinian State Medical University, 42 Kobylyanska st., 58000, Chernivtsi, Ukraine \\ c Department of Physics, Chemistry and Biology, Linköping University, SE-581 83 Linköping, Sweden
}

\section{A R T I C L E I N F O}

Available online 24 April 2013

\section{Keywords:}

Composite

Polymer

CdS nanoparticles

Photoluminescence

Decay

\begin{abstract}
A B S T R A C T
Participation of a polymeric media in light-emitting processes of composite nano-CdS/polyvinyl alcohol is studied by probing different absorption-emission routes via adjustment of excitation wavelengths. It is shown that the polymeric constituent of the composite contributes chiefly to the photoluminescence excitation processes via absorption and excitation transfer to the embedded CdS nanoparticles while the composite emission occurs mostly within the nanoparticles.
\end{abstract}

(C) 2013 Elsevier B.V. All rights reserved.

\section{Introduction}

Polymer/nanoparticles composites are rapidly developing functional materials attractive for a large variety of applications in the fields of photonics, sensorics, biotechnologies, etc. [1]. Polymers, loaded with semiconductor nanoparticles (NPs), acquire additional features obtained from combining intrinsic advantages of polymers with size-dependent properties of NPs. In particular, nanocomposite CdS/polyvinyl alcohol (PVA) is the subject of increasing interest. PVA is a widely used polymer; its numerous applications range from textile and metallurgical industry to medicine and fine biotechnologies [2]. It is colorless, flexible, low-cost, and bio-degradable. This polymer provides a stable matrix to host nanocrystals and, being transparent in the visible range, is suitable for various optical studies and applications. As a part of the composite, CdS NPs allow wavelength tuning of visible light emission that makes the material a promising candidate for applications in light-emitting devices.

In view of the described attractive features, the system CdS/PVA has been extensively studied using photoluminescence (PL) spectroscopy that was proved to be one of the most convenient methods for this purpose; see, e.g. [3,4]. The majority of previous studies were focused on intrinsic PL of NPs while only few papers discussed the participation of the host polymer in the processes of light emission and excitation $[5,6]$. However, it should be noted that the PVA matrix is not a mere support for NPs. While the intrinsic highest occupied molecular orbital (HOMO)-lowest unoccupied molecular orbital (LUMO) gap of the pure PVA corresponds to the ultraviolet $\mathrm{C}$ range, commercially available materials always contain some amounts of

\footnotetext{
* Corresponding author at: 45, Pr. Nauky, Kiev, 03028, Ukraine. Tel.: + 38445258303. E-mail address: g_rudko@yahoo.com (G.Y. Rudko).
}

radicals and chromophore groups. These imperfections can absorb and emit light in the long-wave ultraviolet A or even in the visible ranges [7-9] and, thus, can contribute to the overall optical properties of the composite.

In the present work we study the participation of the polymer matrix in the PL emission of the CdS/PVA nanocomposite as a whole. For this purpose we have chosen two excitation wavelengths which, on the one hand, provide excitation of either the lowest or very high excited states in NPs, and on the other hand, are absorbed by different chromophore groups in the polymer.

\section{Experimental details}

\subsection{Synthesis of the CdS/PVA composite}

Colloidal CdS NPs were synthesized in a water solution of PVA (with no more than 4 wt.\% of acetate groups, $\mathrm{pH} 5-8$ ) using $\mathrm{CdCl}_{2}$ and $\mathrm{Na}_{2} \mathrm{~S}$ as precursors for particles growth. Molecules of PVA served as capping agents that restricted the size of NPs during the growth. Concentrations of $\mathrm{CdCl}_{2}$ and $\mathrm{Na}_{2} \mathrm{~S}$ salts in the solution, its $\mathrm{pH}$ values, and sequences of synthesis steps can be found in [10]. All synthesis steps were performed under ambient conditions.

The method yielded colloidal solutions of CdS NPs that were further used to form solid nanocomposite films by drying in a pressure-tight vessel that contained an absorbent. The temperature of drying was $300 \mathrm{~K}$.

\subsection{Characterization techniques}

Optical absorption measurements were carried out at $300 \mathrm{~K}$ using a deuterium lamp DDS-30 and a MDR-23 monochromator. PL emission 
was excited by a mode-locked Ti:sapphire pulsed laser that was operated at two wavelengths $-250 \mathrm{~nm}(4.96 \mathrm{eV})$ and $415 \mathrm{~nm}(2.98 \mathrm{eV})$. The pulse repetition rate was $80 \mathrm{MHz}$. Time-integrated PL spectra as well as luminescence decays were recorded at $200 \mathrm{~K}$ using a streak-camera system equipped with a $0.5 \mathrm{~m}$ monochromator. The response time of the system was about 20 ps. During the PL measurements the samples were kept in chemically inert helium atmosphere. The temperature of measurements was $200 \mathrm{~K}$. This temperature was low enough to prevent temperature quenching of a red PL band of NPs [11].

Transmission electron micrographs (TEM) were recorded using a high resolution FEI Tecnai G2 200 keV FEG instrument. TEM samples were prepared by deposition of colloidal particles suspended in ethanol on 200 mesh copper grid.

\section{Experimental results}

\subsection{Nanoparticles size}

Fig. 1 shows, as an example, the representative transmission electron micrograph of CdS nanoparticles. It is seen that NP sizes are quite dispersed and that the NPs are not spherical. The average diameter of nanoparticles is equal to $5.4 \pm 0.4 \mathrm{~nm}$.

\subsection{Absorption of PVA and CdS/PVA}

Fig. 2 shows absorbance spectra measured within the ultravioletvisible spectral range from the unloaded host polymer, nanocomposite CdS/PVA and single crystalline CdS. All structures have similar thicknesses of $100 \pm 10 \mathrm{mkm}$. It is seen that the HOMO-LUMO absorption edge of PVA lies at much higher energies than the absorption edge of the composite. Keeping in mind that the absorption edge of the composite is predominantly determined by light absorption in $\mathrm{CdS}$ nanoparticles that are embedded in PVA one can state that the polymer matrix presents a confining potential for charge carriers within the CdS NPs (to be discussed below, see Fig. 5). This statement is further supported by the observation that the absorption edge of CdS/PVA lies at higher energies as compared with the one of bulk CdS. These point to the confinement-related increase of the band gap in the semiconductor NPs. Due to the wide dispersion and non-spherical shapes of NPs, no distinct absorption peaks from the CdS NPs can be resolved in the absorbance spectrum of CdS/PVA.

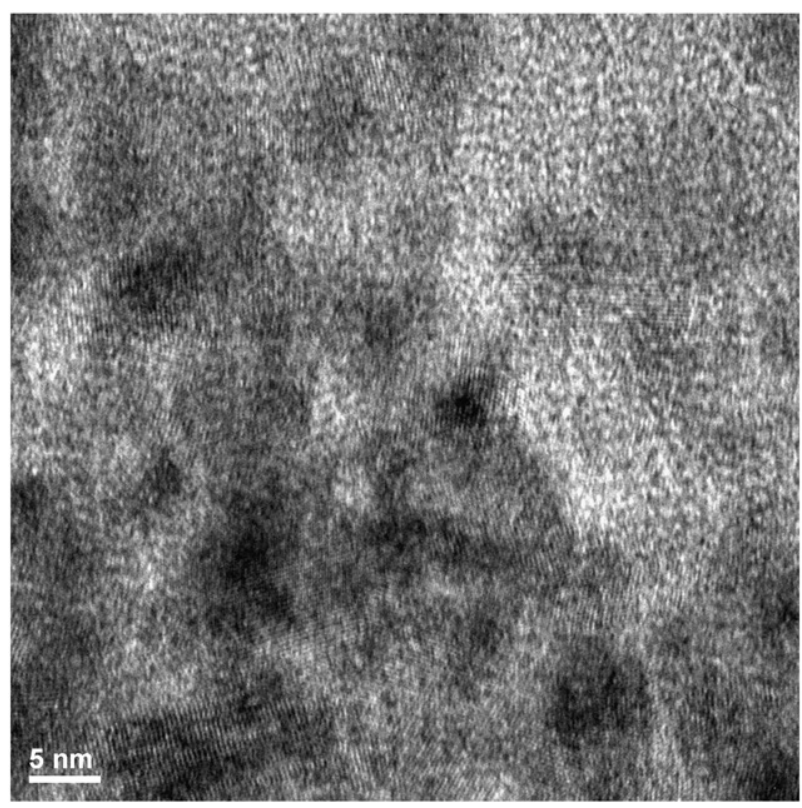

Fig. 1. Transmission electron micrograph of CdS nanoparticles.

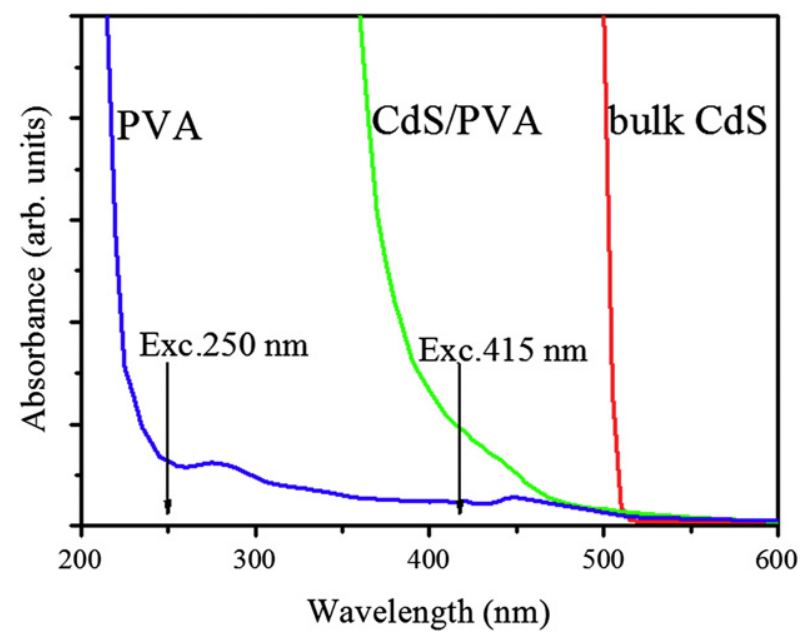

Fig. 2. Optical absorbance spectra of the unloaded PVA, nanocomposite CdS/PVA and bulk CdS crystal. The arrows indicate the wavelengths used for PL excitation.

Two wavelengths, i.e. $250 \mathrm{~nm}$ and $415 \mathrm{~nm}$, were chosen for the PL excitation as marked by the arrows in Fig. 2. The former lies at the high-energy edge of the absorption band of carbonyl groups in the unloaded PVA (centered at $280 \mathrm{~nm}$ [12]) and also corresponds to strong absorption into the higher laying excited states of the CdS NPs. The latter matches absorption transitions between the ground and low laying excited states of the CdS NPs and a weak absorption background of the unloaded PVA.

\subsection{Time-integrated $P L$}

Fig. 3 shows time-integrated PL spectra of PVA (a) and nanocomposite CdS/PVA (b) measured at two excitation wavelengths (415 and $250 \mathrm{~nm}$ ). At both excitations, a single PL band is observed for the unloaded PVA matrix (Fig. 3a). The PL maximum position shifts, however, from $450 \mathrm{~nm}$ to $515 \mathrm{~nm}$ when the excitation wavelength $\left(\lambda_{\text {exc }}\right)$ is changed from $250 \mathrm{~nm}$ to $415 \mathrm{~nm}$. The spectrum of the nanocomposite, on the other hand, consists of two PL bands (Fig. 3b) in the green and red spectral ranges, and no traces of the polymer emission are seen. Spectral positions of both PL bands and their relative intensities do not depend on $\lambda_{\text {exc }}$, which implies that the same radiative centers are responsible for the emission observed at both excitations.

The observed spectrum is quite common for CdS nanocrystals [11,13-17] and has been observed for CdS NPs in various media, such as colloidal solutions, various polymer matrices (including PVA), and glass.

The "green" and "red" PL bands in colloidal nanoparticles are typically ascribed to the emission of shallow [18,19] and deep traps [15], although the exact origin of these bands remains unclear. Our time-decay results (see Fig. 4b, c) support the opinion that these PL bands have different origins because the characteristic PL decay times differ by orders of magnitude. Thus, in what follows we will regard these bands as being from two different sets of levels related to NPs.

\subsection{PL decays}

Fig. 4 shows experimentally measured decays of the PVA emission (a) as well as of the "green" (b) and "red" (c) PL bands in the nanocomposite CdS/PVA (the solid lines). It is seen that, in general, the change of the excitation wavelength causes opposite trends in the PL decays in two materials. Specifically, whereas an average decay rate increases in PVA when the excitation wavelength is changed from 250 to $415 \mathrm{~nm}$, the decays become slower in the CdS/PVA under the same change of $\lambda_{\text {exc }}$. It should be noted that the shape of the PL bands both in PVA and CdS/PVA does not change during the decay. 


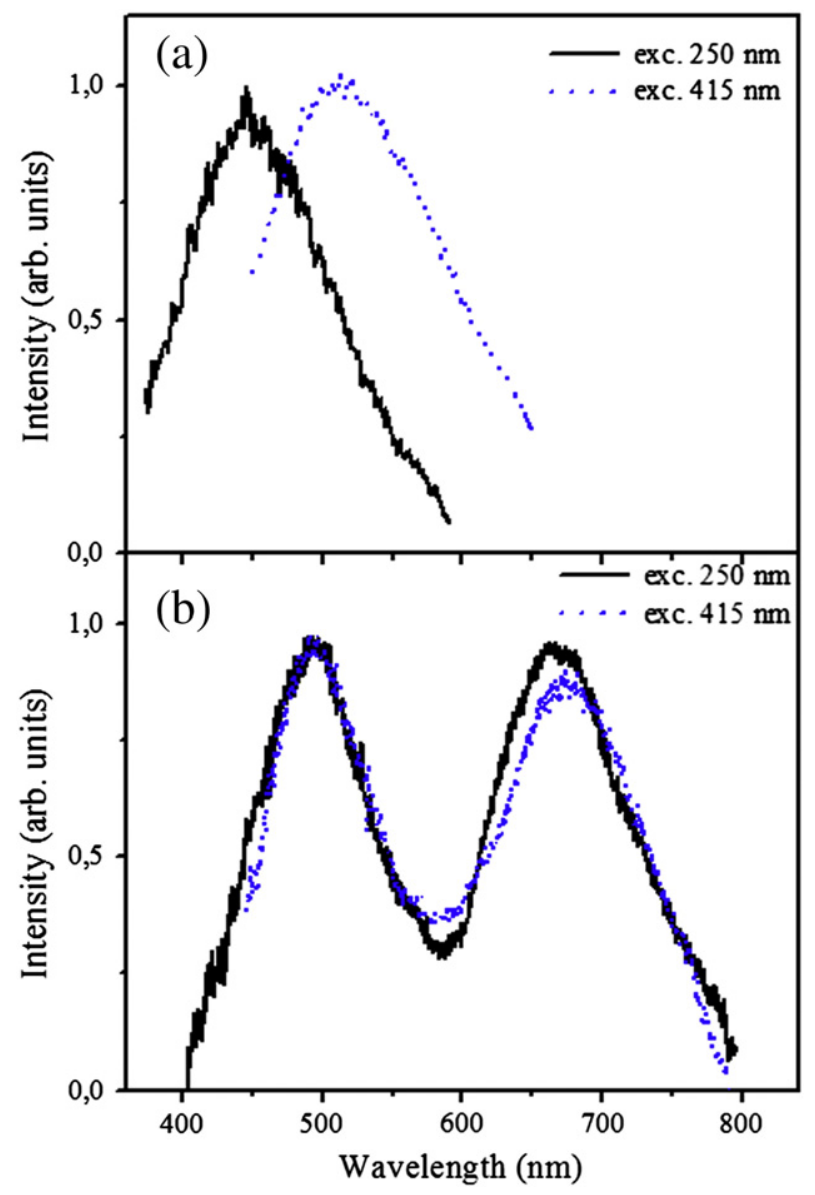

Fig. 3. Time-integrated PL spectra of the unloaded PVA (a) and CdS/PVA (b). The spectra are normalized to the same peak intensity, $T=200 \mathrm{~K}, P=5 \mathrm{~mW}$.

More detailed analysis demonstrates that the decay curve of the unloaded PVA measured at $\lambda_{\text {exc }}=250 \mathrm{~nm}$ is mono-exponential with a characteristic time of $4.4 \pm 0.1 \mathrm{~ns}$, as shown by the dashed line in Fig. 4a. The transient behavior of PVA at $\lambda_{\text {exc }}=415 \mathrm{~nm}$ has a multi-exponential character. An average decay time of this multiexponential process estimated as the time when the PL intensity decreases by $e$ times is equal to $1.0 \pm 0.1 \mathrm{~ns}$.

The decay curves for the "green" band of CdS/PVA can be fitted (dashed lines) by bi-exponential functions in the form $I(t)=$ $a_{1} e^{-t / \tau 1}+a_{2} e^{-t / \tau 2}$, where $I(t)$ is the time-dependent PL intensity, and $\tau_{1}$ and $\tau_{2}$ are the lifetimes of two components with relative intensities of $a_{1}$ and $a_{2}$. For $\lambda_{\text {exc }}=250 \mathrm{~nm}$ the best fit to the experimental data was obtained by using the following parameters: $\tau_{1}=0.60 \pm 0.03 \mathrm{~ns}$, $\tau_{2}=8.5 \pm 0.4 \mathrm{~ns}, a_{1}=0.90 \pm 0.05$, and $a_{2}=0.23 \pm 0.01$. The characteristic decay times remain practically the same when $\lambda_{\text {exc }}$ was tuned to $415 \mathrm{~nm}$, i.e. $\tau_{1}=0.60 \pm 0.03 \mathrm{~ns}$, and $\tau_{2}=8.1 \pm 0.4 \mathrm{~ns}$. On the other hand, the prefactors $a_{1}$ and $a_{2}$ need to be changed to $a_{1}=$ $0.60 \pm 0.03$, and $a_{2}=0.66 \pm 0.04$. Thus, different transient behavior of CdS/PVA at two excitations is mainly caused by different contributions of the fast and slow decay processes to the overall decay and the relative contribution of the slow processes is larger when $\lambda_{\mathrm{exc}}=415 \mathrm{~nm}$.

The "red" band of CdS/PVA (Fig. 4c) exhibits a very long decay. The estimated decay time is by orders of magnitude longer than the one for the "green" band. Therefore no changes can be detected with our experimental setup under the variation of $\lambda_{\text {exc }}$ and only one decay curve is presented in Fig. 4c. This emission will not be further discussed in the paper.

\section{Discussion}

The obtained results allow us to propose a detailed scheme of the absorption and emission transitions in the unloaded PVA and CdS/PVA composite as shown schematically in Fig. 5. In this scheme positions and widths of energy levels, as well as lengths of the arrows which indicate the excitation and PL processes are scaled in accordance with reported and our experimental data. The energy position of the LUMO of PVA relative to the vacuum level is chosen in accordance with [20]. The valence band edge of bulk CdS is depicted based on an electron affinity value taken from [21]. The energy position of the conduction band edge of CdS is shifted by $\sim 0.34 \mathrm{eV}$ to the higher energy as compared with that in bulk CdS, to account for the experimentally observed shift of the absorption edge in CdS NPs due to quantum confinement effects. The scatter of this state energy due to the statistical distribution of NPs sizes is ignored. Quantization of holes energy is neglected since the effective mass of hole in CdS is $\sim 4$ times larger than that of electron. The justification for this model is given.

In the unloaded PVA (Fig. 5a) the absorption of light with $\lambda_{\mathrm{exc}}=$ $250 \mathrm{~nm}$ causes the transitions between HOMO and LUMO states of carbonyl groups and gives rise to the emission band characterized by a large Stockes shift. The decay of this emission is single-exponential (Fig. 4a) which points to the participation of only one type of centers in the radiative recombination process, presumably, carbonyl-related chromophore groups [22].

Excitation light with $\lambda_{\text {exc }}=415 \mathrm{~nm}$ is absorbed by the unloaded PVA within very weak absorption continuum (Fig. 2). According to K. Maruyama [9] the only irregular chain fragments of PVA that have electronic transitions in this spectral range are polyenes. However, due to very low concentration of the species responsible for the absorption in this range it was not possible to unambiguously identify them. Despite the weak absorption, the excitation at $\lambda_{\mathrm{exc}}=415 \mathrm{~nm}$ produces

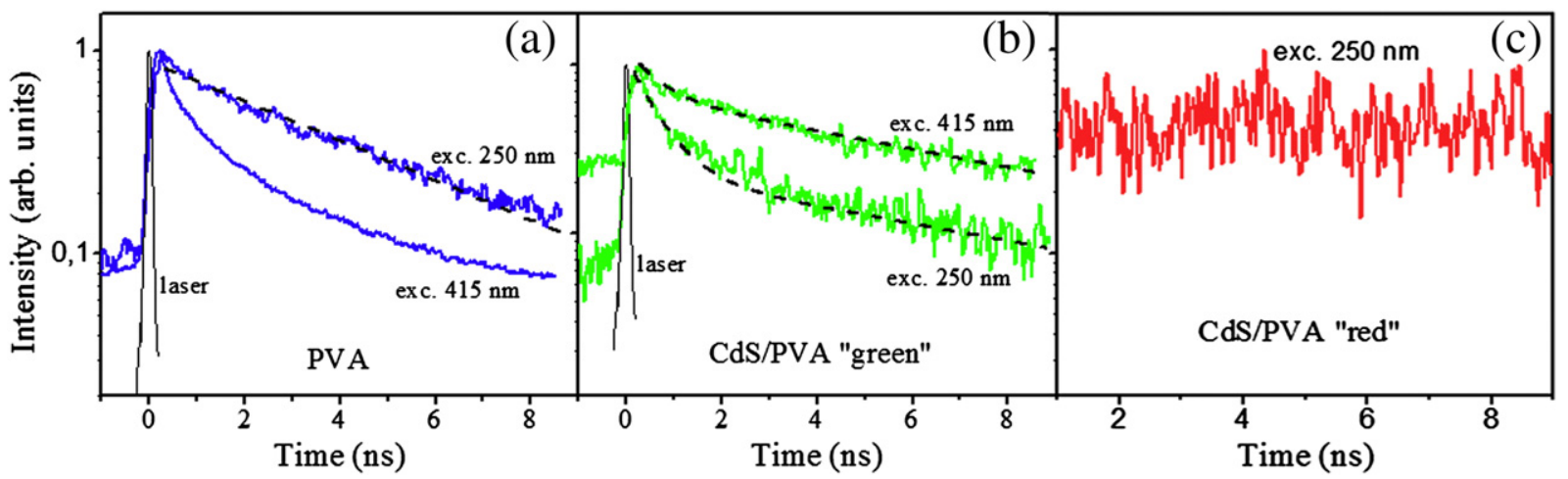

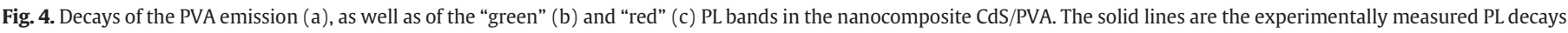

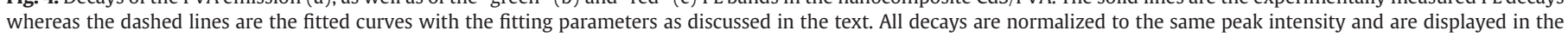
semi-logarithmic scale. 


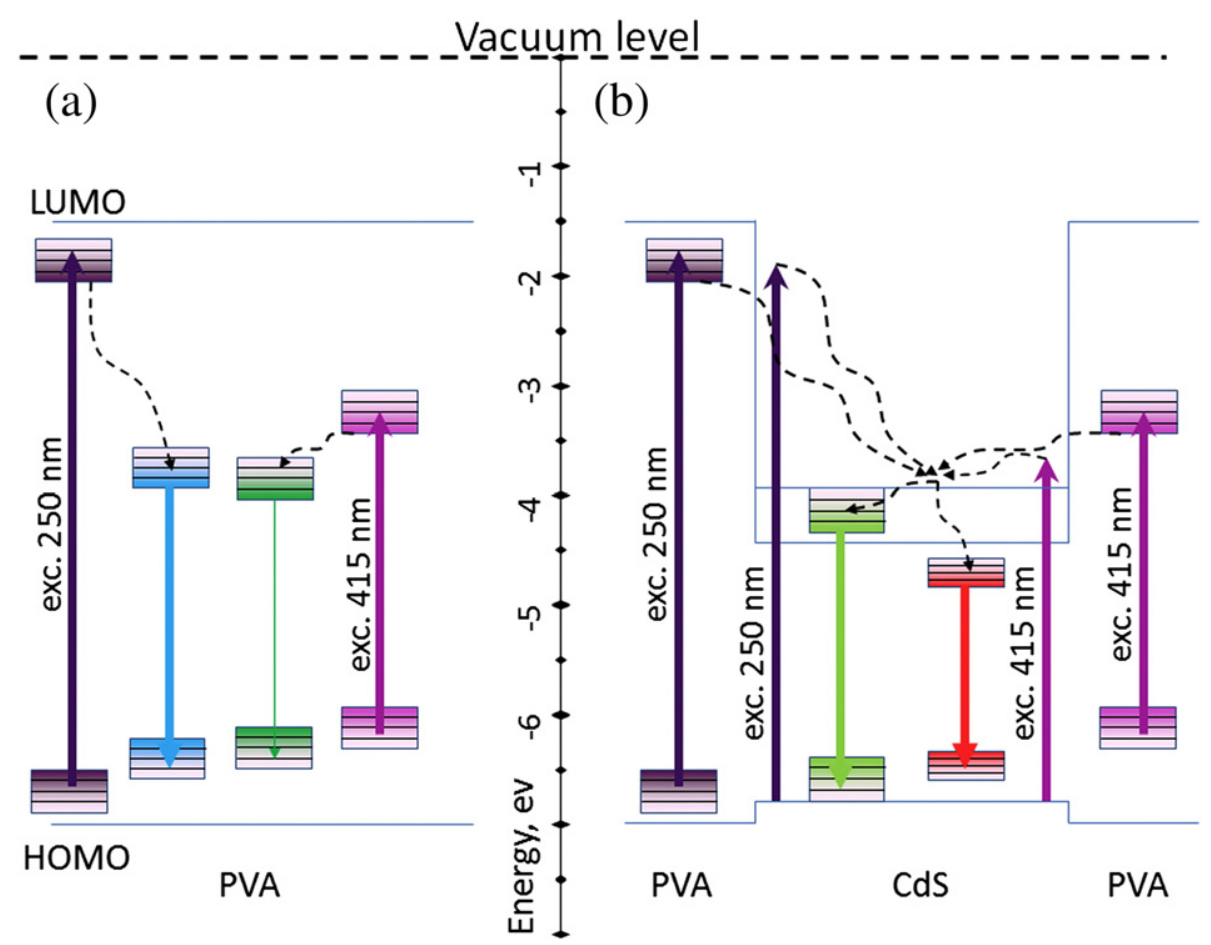

Fig. 5. Energy diagram of the transitions in PVA (a) and CdS/PVA composite (b).

the intense PL band in PVA. This PL band exhibits multiexponential decay that likely reflects participation of many different light-emitting species.

In the CdS/PVA composites the absorption and emission processes (Fig. $5 \mathrm{~b}$ ) are defined by both constituents of the composite and their interactions. The absorption of light with $\lambda_{\text {exc }}=250 \mathrm{~nm}$ occurs both in the matrix and in CdS NPs (transitions to very high excited states). Light with $\lambda_{\text {exc }}=415 \mathrm{~nm}$, on the other hand, can cause both the excitation of absorbing species in PVA and the transitions to the lower-lying excited levels within CdS NPs (Fig. 5b). Taking into account that no polymer-like emission is seen in the PL spectra of the composite (Fig. 3b) one can conclude that the light-emitting processes occur predominantly within the CdS nanoparticles, i.e. that the NPs provide the alternative de-excitation channel for the matrix.

Excitation-emission processes in the composite can be further understood by analyzing decays of the "green" emission measured under different $\lambda_{\text {exc }}$. We note, that based on the results of the performed absorption measurements (Fig. 2), the fraction of light absorbed within the CdS NPs depends on $\lambda_{\mathrm{exc}}$ and is higher for $\lambda_{\mathrm{exc}}=250 \mathrm{~nm}$. It is, therefore, reasonable to assume that the decay of the "green" emission measured under $250 \mathrm{~nm}$ excitation will be largely determined by the intrinsic absorption and emission processes that occur solely within the CdS NPs. From Fig. 3a, these processes should be responsible for the faster PL decay with $\tau_{1} \approx 0.6$ ns. The second component of the PL decay can then be interpreted as a result of excitation transfer from the polymeric matrix to NPs. Due to this external feeding, the emission of NPs lasts longer and decays with the characteristic time $\tau_{2} \approx 8.1-$ $8.5 \mathrm{~ns}$. An efficiency of the transfer itself may also change with $\lambda_{\text {exc }}$. For example, if the transfer requires initial energy relaxation, some of the excitation energy may be lost via non-radiative recombination. These losses are expected to be higher for shorter excitation wavelengths when a larger portion of the excitation energy must be dissipated within PVA.

Different transfer mechanisms can be discussed as a possible origin of the excitation of NPs via the polymer matrix. One is the drain of photogenerated excitons from the polymer to the nanoparticles. This mechanism is quite possible in the proposed energy scheme (Fig. 5). Indeed, since the energy levels of the CdS NPs lie within the HOMO-LUMO gap of the polymer they can readily capture both photoexcited electrons and holes. Another mechanism is energy transfer due to either re-absorption or dipole-dipole interaction of the Forster type [23]. In the case of $\lambda_{\mathrm{exc}}=250 \mathrm{~nm}$ the condition of the Forster-type transfer is easily fulfilled since there is a spectral overlap between the emission band of the energy donor (polymer matrix) and the absorption band of the energy acceptor (NP). However, at $\lambda_{\mathrm{exc}}=415 \mathrm{~nm}$ the spectral overlap is reduced which could make the transfer less efficient. To distinguish among all possible mechanisms of energy transfer further studies are necessary and are currently in progress.

\section{Conclusions}

Participation of the polymer matrix in the light absorption and emission processes of the CdS/PVA composite is analyzed employing the time-resolved PL spectroscopy. We show that decays of the PL emission from the CdS NPs embedded in the composite critically depend on the excitation wavelength. This behavior is ascribed to the interplay between the intrinsic NPs excitation and extrinsic feeding of the NPs via energy transfer from the excited polymer matrix. Possible mechanisms of the observed energy transfer are also discussed.

\section{Acknowledgements}

Financial support from the Swedish Institute via Visby program is greatly appreciated.

\section{References}

[1] P. Reiss, E. Couderc, J. De Girolamo, A. Pron, Nanoscale 3 (2011) 446.

[2] K.M.L. Taylor-Pashow, J.D. Rocca, R.C. Huxford, W. Lin, Chem. Commun. 46 (2010) 5832.

[3] D.S. Yoo, S.Y. Ha, I.G. Kim, M.S. Choo, K.W. Kim, E.S. Lee, New Phys. Sae Mulli 61 (7) (2011) 680.

[4] H.S. Mansur, A.P. Mansur, J.C. González, Polymer 52 (2011) 1045

[5] Y. Badr, M.A. Mahmoudb, Spectrochim. Acta A 65 (2006) 584

[6] R. Seoudi, S. Abd El Mongy, A.A. Shabaka, Physica B 403 (2008) 1781.

[7] J. Mieloszyk, R. Drabent, J. Siodmiak, J. Appl. Polym. Sci. 34 (1987) 1577.

[8] L.V. Smirnov, N.V. Platonova, N.P. Kulikova, J. Appl. Spectrosc. 8 (2) (1968) 308

[9] K. Maruyama, Y. Kuramoto, Y. Tanizaki, Polymer 30 (1989) 1419.

10] G.Yu. Rudko, A.O. Kovalchuk, V.I. Fediv, J. Beyer, W.M. Chen, I.A. Buyanova, Adv. Sci. Eng. Med. 4 (5) (2012) 394 
[11] A. Eychmuller, A. Hasselbarth, L. Katsikas, H. Weller, Phys. Chem. 95 (1) (1991) 79.

[12] S.P. Vijayalakshmi, G. Madras, J. Appl. Polym. Sci. 102 (2006) 958.

[13] D. Kim, T. Mishima, M. Nakayama, Physica E 21 (2004) 363.

114] Y. Ohara, T. Nakabayashi, K. Iwasaki, T. Torimoto, B. Ohtani, N. Ohta, C. R. Chim. 9 (2006) 742.

[15] M. Tanaka, J. Qi, Y. Musumoto, J. Cryst. Growth 214 (215) (2000) 410.

16] K. Tomihira, D.G. Kim, M. Nakayama, J. Lumin. 122 (2007) 471.

[17] B.G. Potter Jr., J.H. Simmons, Phys. Rev. B 37 (1988) 10838.
[18] S. Santhi, E. Bernstein, Francoise Paille, J. Lumin. 117 (2006) 101.

[19] G. Ma, S.-H. Tang, W. Sun, Z. Shen, W. Huang, J. Shi, Phys. Lett. A 299 (5-6) (2002) 581.

[20] S.P. Mondal, V.S. Reddy, S. Das, A. Dhar, S.K. Ray, Nanotechnology 19 (2008) 215.

[21] S. Adachi, John Wiley \& Sons, Ltd, Chichester, 2005.

[22] M. Konishi, T. Isobe, M. Senna, J. Lumin. 93 (2001) 1.

[23] T. Forster, Ann. Phys. 55 (1948) 2. 